\title{
312 - Level of engagement during an innovative therapy program in people with dementia - implementing Serious Traditional Multidimensional Games.
}

Marlene C. Neves Rosa, Sr., PhD; Raul Antunes; Rui Matos; Nuno Amaro; Marta Duarte

\section{Key words - Games, therapy, dementia, engagement}

Background: There is a significant difficulty in the engagement of people with dementia in therapeutic activities. Considering that stimulus attributes (e.g., content of a specific activity) seem to be determinant to achieve an expected engagement, innovative approaches are required. Aim: characterise the engagement of people with dementia in serious traditional multidimensional games (sTMG), comparing with conventional therapy (CT) sessions. Methods: Subjects with dementia were recruited in Alzheimer's Portugal Foundation. Sociodemographic and clinical participants' characteristics were collected, including classification of dementia severity using Mini-mental State Examination (MSE) and walking independence classification (dependent - someone needs supervision or human support to walk). Gerontologist, psychologist, occupational therapist and physiotherapist were invited to classify patients' engagement in routine CT (i.e., cognitive therapy and exercise classes), according to 0-10 scale (10 - successful engagement). Serious adaptations in TMG were implemented in 3 consecutive sessions (1 per week/ $45 \mathrm{~min} . /$ session) and patient s engagement was rated in each session. Success index (number of subjects showing higher engagement in TMG/total participants) was calculated. Results: Thirteen participants (5 males; 79.23 $\pm 8.39 y r s ; 15.76 \pm 9.22 \mathrm{MSE} ; 9$ walk independently) were enrolled. Success index was $38 \%$; comparing TMG with cognitive therapy and $31 \%$, comparing to movement classes. Two patients with severe dementia and walking independency were more engaged in sTMG sessions (sTMG - 4;4; Cognitive therapy - 2;1; Exercises Classes). Four patients with moderate dementia and walking independency obtained worse engagement (sTMG -3;6;2;7; Cognitive therapy - 6;7;8;8 Exercises Classes $-4 ; 6 ; 7 ; 9)$. Discussion: Our results showed that sTMG sessions had a positive impact in people with dementia, specifically in advanced/severe cases. In this respect, a previous study of Natalie et al., (2017) concluded that engagement was lower in cognitive activities for people with severe dementia, which can explain the higher engagement in sTMG sessions. Furthermore, Bier et al. (2008) enlightened that people with dementia in a moderate stage are characterised by emergent behavioural changes, which might difficult patients' integration in new activities. Conclusion: sTMG had a positive impact in the engagement of people with dementia. Specifically, for patients at middle stage of dementia, future studies include longer sTMG protocols, possibly providing better patients' integration. 